\title{
Clinical utility of morphology, immunohistochemistry, flow cytometry, and FISH analysis in monitoring of plasma cell neoplasms in the bone marrow
}

\author{
Oluyomi E. Ajise ${ }^{1,2} \cdot$ Mikhail Roshal $^{1} \cdot$ Lu Wang $^{1} \cdot$ Goutamie N. Sukhram $^{1}$. \\ Katherine M. Smith ${ }^{1} \cdot$ Peter Maslak $^{1} \cdot{\text { Ahmet } \operatorname{Dogan}^{1}}^{1}$
}

Received: 15 September 2015 / Accepted: 8 December 2015/Published online: 23 December 2015

(C) Springer-Verlag Berlin Heidelberg 2015

\begin{abstract}
In this study, we examined clinical utility of morphology, immunohistochemistry (IHC), flow cytometric immunophenotyping (FC), and multiple myeloma (MM)-specific fluorescence in situ hybridization (FISH) in evaluation of disease burden in bone marrow (BM) of plasma cell neoplasms (PCNs). Of the 100 consecutive BM samples, 81 demonstrated PCN. CD138 IHC was the most sensitive technique to assess PCN burden compared to aspirate count or flow cytometry with mean values of $30 \%$ (range 3-100), 19 (178), and 2.1 (0.01-33.2), respectively. CD138 IHC correlated well with the aspirate count $\left(r^{2}=0.5\right)$ but poorly with FC $\left(r^{2}=0.1\right)$. FC demonstrated the highest sensitivity in assessment of clonality $96 \%$ (79/82), while morphology and IHC was positive in $84 \%$ (71/82), FISH in $79 \%$ (59/75) cases. Our results show that $\mathrm{CD} 138 \mathrm{IHC}$ is the most sensitive method for assessment of BM PCN burden whereas FC is the most sensitive method to establish the clonal nature of the PCN.
\end{abstract}

Keywords Bone marrow $\cdot$ Plasma cell myeloma $\cdot$ Flow cytometry $\cdot$ Immunohistochemistry $\cdot$ FISH

\section{Introduction}

Plasma cell myeloma/multiple myeloma (MM)) is a malignancy of terminally differentiated plasma cells (PCs) and is

Ahmet Dogan

dogana@mskcc.org

1 Memorial Sloan Kettering Cancer Center, 1275 York Avenue, New York, NY 10065, USA

2 Present address: Department of Pathology, McGill University, Montreal, Quebec H3A 2B4, Canada the second most common hematologic neoplasm after lymphoma [1]. MM comprises about $1 \%$ of malignant tumors and $10-15 \%$ of hematopoietic neoplasms and causes $20 \%$ of deaths from hematological malignancies [2]. The detection of cytogenetic abnormalities is essential in risk stratification of MM [3] and is recommended by the best practice guidelines [4-6].

In addition to other serological and clinical criteria, disease diagnosis and monitoring depends on the assessment of PC numbers/burden by morphology and/or immunophenotyping in bone marrow (BM) specimens. According to the International Uniform Response Criteria for Multiple Myeloma, the diagnosis of MM requires the presence of an M-component in serum and/or urine plus clonal plasma cells in the bone marrow and/or a documented plasmacytoma and at least one of the CRAB criteria [7]. In addition, patients with biopsy-proven amyloidosis and/or systemic light chain deposition disease (LCDD) are classified as "myeloma with documented amyloidosis" or "myeloma with documented LCDD, " respectively, if they have greater than or equal to $30 \%$ plasma cells and/or myeloma-related bone disease [7, 8].

Similarly, the assessment of neoplastic plasma cell numbers is an important criterion for disease monitoring. Various clinical therapeutic response categories in patients with $\mathrm{MM}$ require documentation of plasma cell burden in the marrow. The assessment "stringent complete response" requires the absence of clonal plasma cells in the bone marrow as demonstrated by immunohistochemistry and/or flow cytometry; the assessment of "complete response" requires $\leq 5 \%$ plasma cells in the marrow, and the assessment of partial response in patients with unmeasurable serum and urine M-protein and serum-free light assays but with $\geq 30 \%$ plasma cells at diagnosis requires $50 \%$ reduction in plasma cells.

Despite the important role that plasma cell counts/clonality play in the diagnosis and management of myeloma, very few 
studies have addressed the utilization of various laboratory methods in establishing plasma cell burden in the marrow. The aim of this retrospective review study is to provide a comparison of different testing modalities such as trephine bone marrow biopsy combined with CD138 and immunoglobulin light chain immmunohistochemical staining, aspirate plasma cell counts, flow cytometric analysis, and MMspecific fluorescence in situ hybridization (FISH) analysis in the assessment of plasma cell burden.

\section{Material and methods}

\section{Patients and samples}

The study was approved by the Institutional Review Board at Memorial Sloan Kettering Cancer Center (MSKCC). One hundred consecutive patient samples submitted for evaluation of a plasma cell neoplasm/myeloma were identified using a search of the surgical pathology database. Patient records were accessed for age at diagnosis, sex, new or previously established disease, final diagnosis, percentage of plasma cells on biopsy combined with immunohistochemistry (BM/IHC), aspirate, and flow. In addition, FISH analysis for myelomaspecific chromosomal abnormalities, serum-free light chains, immunofixation, and beta 2 microglobulin records was noted.

\section{Immunohistochemical analysis}

Sections of formalin-fixed paraffin-embedded bone marrow biopsy $(5 \mu \mathrm{m}$ thick) were stained with routine clinical methods using monoclonal antibodies specific for CD138, kappa, and lambda A (all from Leica Biosystems, Newcastle, UK). All IHC staining were performed in the Bond system automatic immunostaining device using a bond polymer refine detection kit (Leica Biosystems). Heatmediated antigen retrieval was carried out in all samples. Tonsil tissue was used as positive controls, while negative controls were performed by using a negative monoclonal antibody on the patient samples. Immunostained sections were counterstained with hematoxylin.

Plasma cell burden was assessed based on the percentage of CD138-positive plasma cells as compared to all nucleated bone marrow elements using estimates with $5 \%$ increments as previously described [9]. The assessment was performed as part of standard clinical care, and the results were obtained from pathology reports. Morphologic evaluation was judged positive for a plasma cell neoplasm when greater than $5 \%$ plasma cells with kappa or lambda light chain restriction were present in the bone marrow biopsy. For kappa light chain restriction, a ratio greater than 5:1 $=\mathrm{kappa} / \mathrm{lambda}$ and, for lambda light chain restriction, a ratio lower than 1:2=kappa/ lambda was used. All 100 samples had an evaluable biopsy.

\section{Aspirate smear preparation}

Bone marrow first-pull aspirate smears stained with tetrachrome/Wright Giemsa were used for morphologic assessment. The assessment was performed as part of standard clinical care, and the results were obtained from pathology reports. A 400 cell differential count was obtained using conventional bright-field microscopy. Ninety two of the 100 samples in this study had an evaluable aspirate smear. Two samples had poor aspirate quality, while six did not have a concurrent aspirate smear performed. Morphologic evaluation was judged positive for a plasma cell neoplasm when greater than $5 \%$ PC was present in the BM aspirate sample based on previously established consensus criteria [8].

\section{Flow cytometric analysis}

Immunophenotypic studies were performed on erythrocytelysed (BD Pharm Lyse ${ }^{\mathrm{TM}}$ ) BM aspirate samples within $24 \mathrm{~h}$ of the procedure, using a four-color direct immunofluorescence technique aimed at identifying, quantifying, and characterizing PC. The following antibody combinations were studied as follows: (FITC/PE/PerCPCy5.5/APC: CD19/ CD138/CD38/CD45, CD56/CD138/CD38/CD45, CD20/ CD117/CD38/CD45, CD28/CD33/CD38/CD45, KAPPA/ LAMBDA/CD38/CD45, cyKAPPA/-/CD38/CD45, and cyLAMBDA/-/CD38/CD45). All antibodies were purchased from BD Biosciences, San Jose, CA, USA.

Multiparameter flow cytometry (MFC) was performed and analyzed using a FACSCanto ${ }^{\mathrm{TM}}$ flow cytometer (BD Biosciences) and the FACSDiva software. At least 100,000 total events were acquired, and analysis was performed on the bright $\mathrm{CD} 38+$ events and classified as $\mathrm{CD} 45+$ or CD45 - . Flow cytometric $(\mathrm{FC})$ positivity required demonstration of at least 50 LC-restricted PCs showing abnormal immunophenotype. An abnormal immunophenotype was determined to be due to a lack of expression of CD19 and/or expression of CD20, aberrant expression of CD28, CD56, or $\mathrm{CD117}$, and/or light chain restriction $\mathrm{k} / \lambda$ of $>4: 1$ or $<1: 2$. All 100 samples had a corresponding FC analysis performed.

\section{Fluorescence in situ hybridization analysis}

Interphase FISH analysis was done on specimens after CD138 cell enrichment using a magnetic bead-based cell isolation method (STEMCELL Technologies Inc., Vancouver, BC, Canada). Before enrichment, a cell count was performed using a Coulter Counter. CD138 cell enrichment was done following the manufacturer's protocol. Briefly, the bone marrow sample was diluted with a 1:1 volume of EasySep Lysis Buffer. EasySep positive (CD138) selection cocktail was added to the bone marrow/lysis buffer mixture and incubated at room temperature for $15 \mathrm{~min}$. EasySep magnetic 
nanoparticles were then added to the bone marrow/lysis buffer mixture and incubated at room temperature for $10 \mathrm{~min}$. The cell pellet was resuspended in a RoboSep DNase I buffer. This automated process was programmed as per the manufacturer's instructions. Tubes were then centrifuged at 1200 revolutions per minute (rpms) for $10 \mathrm{~min}$, and cell counts were again assessed. After careful removal of the supernatant, the pellet was diluted in a hypotonic potassium chloride solution and placed in a $37^{\circ} \mathrm{C}$ incubator for $30 \mathrm{~min}$, followed by the routine methanol-acetic acid (3:1) fixation. Fixed cell pellets were ready for FISH analysis.

The number of FISH probes applied to samples depended on the amount of plasma cells obtained from the EasySep procedure. Our FISH analysis starts with the NCCNrecommended markers, i.e., IGH fusions, gain of $1 \mathrm{q} 21$ [1p32/1q21 probe set], deletion of $17 \mathrm{p} 13$ [TP53 probe], deletions of the long arm of chromosome 13 (13q-)[13q14/13q34 probe set], followed by other markers commonly associated with $\mathrm{MM}$, i.e., gain of 11/11q [MLL probe], deletions of the short arm of chromosome 12 (12p-) [TEL probe], 16q deletion $[C B F B$ probe]. If all the aforementioned markers were negative, the low-risk markers, i.e., trisomy 5, 9, and 15, were performed. In terms of IGH fusions, the analysis started with the high-risk marker, $\mathrm{t}(4 ; 14) / I G H-F G F R 3$ and MMSET hybrid. If this was negative for $\mathrm{t}(4 ; 14)$ but indicating other $I G H$ fusions, we then used FISH for $\mathrm{t}(11 ; 14) / I G H-C C N D 1$, $\mathrm{t}(14 ; 16) / I G H-M A F$, and $\mathrm{t}(14 ; 20) / I G H-M A F B$ for $I G H$ at specific fusions.

\section{Results}

\section{Patient population}

The 100 samples submitted for assessment of plasma cell neoplasm were from a total of 91 patients. Nine patients had a repeated sample submitted for evaluation. The demographic and clinical features of the group are shown in Table 1. The bone marrow studies were performed for diagnosis of PCN in 7 cases and for monitoring of previously diagnosed PCN in 93 cases. A bone marrow biopsy specimen and flow cytometric studies were available in every case, whereas aspirate smears and FISH results were available in 92 and 86 cases, respectively (Table 2). Overall evidence for an abnormal clonal plasma population was identified in 82 cases by flow cytometry and/or immunohistochemistry.

\section{Assessment of plasma cell burden by morphology and immunohistochemistry}

In assessment of the plasma cell burden, CD138 immunohistochemistry with immunoglobulin light chain staining on the biopsy core was the most sensitive method yielding higher
Table 1 Patient demographics and baseline characteristics submitted for the evaluation of PCN

\begin{tabular}{ll}
\hline Male/female (\%) & $58(64 \%) / 33(36 \%)$ \\
Age, years; median (range) & $58(34-81)$ \\
Initial biopsy sample (\%) & $7(7 \%)$ \\
$\begin{array}{l}\text { Follow-up sample (\%) } \\
\text { Clinical diagnosis on submission (\%) }\end{array}$ & $93(93 \%)$ \\
Multiple myeloma & $95(95 \%)$ \\
Amyloidosis & $3(3 \%)$ \\
MGUS & $2(2 \%)$ \\
Transplantation status (\%) & $14(14 \%)$ \\
Post-transplant & $86(86 \%)$ \\
Pre-transplant & \\
Laboratory values (units) & $(1.1-19.5) 2.5$ \\
$\beta 2$ microglobulin, units, median (range) & \\
\hline
\end{tabular}

estimates of the plasma cell numbers $(p<0.01)$ compared to the aspirate count with mean values of $30 \%$ (range 3-100) and $19 \%(1-78)$, respectively. (Table 2, Figs. 1 and 2). Overall, there was a good correlation between CD138-based assessment and aspirate plasma cell counts ( $r$-squared value 0.5, Fig. 3).

\section{Assessment of plasma cell burden by flow cytometric immunophenotyping}

CD138 stain of the biopsy core yielded the highest estimates of the plasma cell proportion $(p<0.01)$ when compared to counts obtained by flow cytometric analysis with mean values of $30 \%$ (range 3-100) and $2.1(0.01-33.2$ ), respectively (Table 2, Figs. 2, 4, and 5). The biopsy proportion estimate correlated rather poorly with flow cytometric evaluation $(r-$ squared value 0.1) (Fig. 6). Flow cytometry demonstrated the highest sensitivity in assessment of clonality $96 \%$ (79/

Table 2 Comparison of the various sample findings when using different modalities

\begin{tabular}{|c|c|c|}
\hline Modality & Normal result (\%) & Abnormal result $(\%)$ \\
\hline $\begin{array}{l}\text { Plasma cells by CD138 IHC } \\
n=100\end{array}$ & $29(29 \%)$ & $71(71 \%)$ \\
\hline $\begin{array}{l}\text { Plasma cells by aspirate counts } \\
n=92\end{array}$ & $33(36 \%)$ & $59(64 \%)$ \\
\hline $\begin{array}{l}\text { Plasma cells by flow } \\
n=100\end{array}$ & $21(21 \%)$ & $79(79 \%)$ \\
\hline $\begin{array}{l}\text { Plasma cells by FISH analysis } \\
n=86\end{array}$ & $27(31 \%)$ & $59(69 \%)$ \\
\hline
\end{tabular}

$n$ total number of samples analyzed by the specific modality 
Fig. 1 a A case of minimal involvement by PCs (black arrow) hematoxylin and eosin, 400 (upper left). b CD138 immunostain performed on BM biopsy of the sample from a showing $5 \%$ involvement (middle left). c The aspirate from the sample demonstrates $4 \%$ PCs tetrachrome/Wright Giemsa, 1000 (lower left). d Focal clusters of PCs from a different patient sample (thick black arrow) hematoxylin and eosin, 400 (upper middle). e CD138 immunostain performed on $\mathrm{BM}$ biopsy of the sample from $\mathbf{d}$ showing $25 \%$ involvement (middle). $\mathbf{f}$ The aspirate from the sample demonstrates $11 \%$ PCs tetrachrome/Wright Giemsa, 1000 (lower middle). $\mathbf{g}$ Sheets of PCs hematoxylin and eosin, 400 (upper right). h CD138 immunostain performed on BM biopsy of the sample from $\mathbf{g}$ showing $80 \%$ involvement (middle right). i The aspirate from the sample demonstrates $59 \%$ PCs tetrachrome/Wright Giemsa, 1000 (lower left)

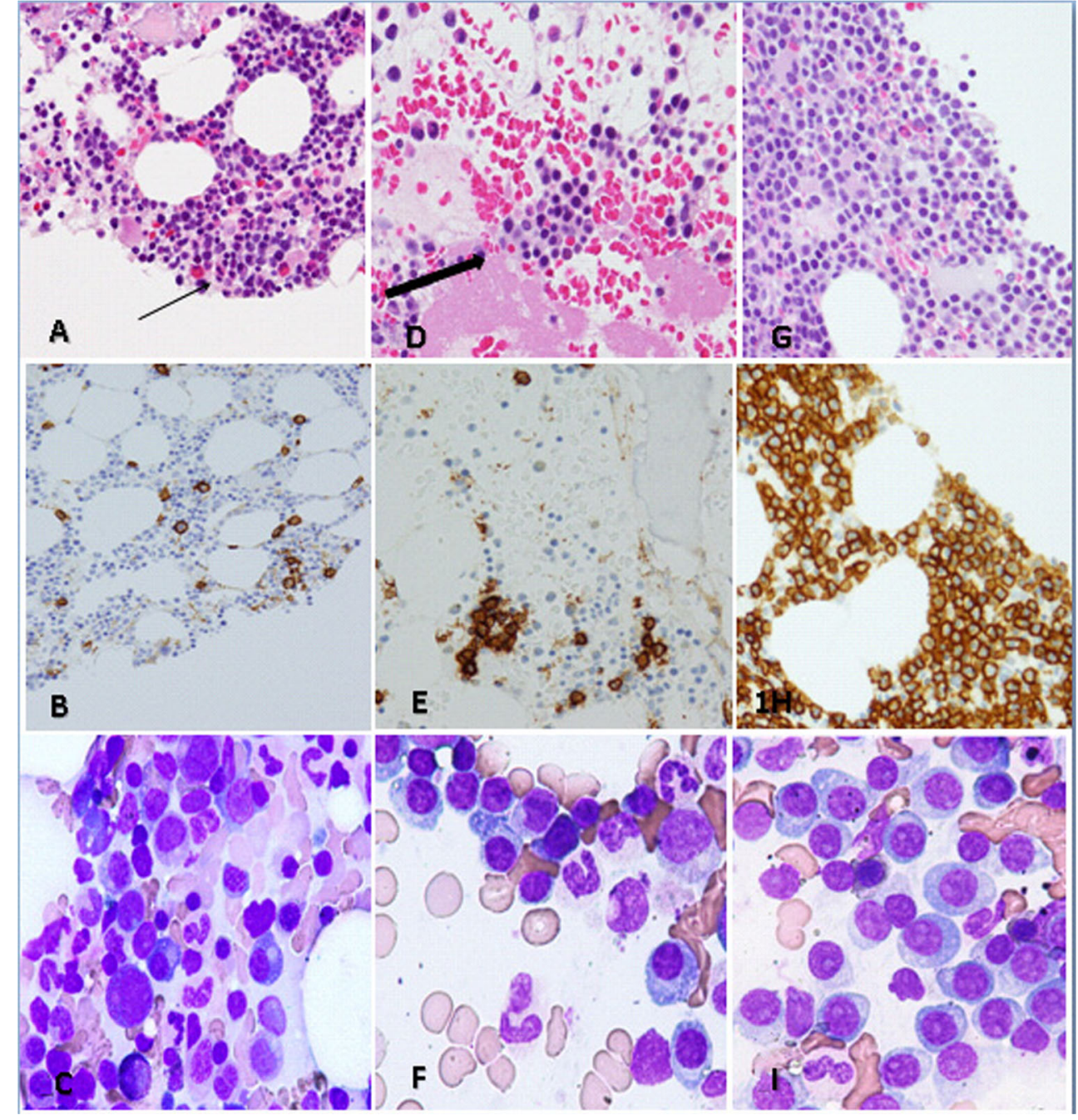

82), while morphology with IHC was judged positive in $84 \%$ (71/82) of cases, followed by MM FISH/cytogenetics $79 \%$ (59/75).

All 79 samples with abnormal flow cytometry results had at least one immunophenotypic aberrancy other than light chain restriction. The most frequent immunophenotypic aberrations were as follows: the absence of CD19 (98\%), CD45 low to negative (88\%), CD56 positivity (69\%), CD117 positivity (43\%), and CD20 positivity (30 \%). In addition, flow cytometry provided information on the proportion of the abnormal plasma cells among the total plasma cell population.

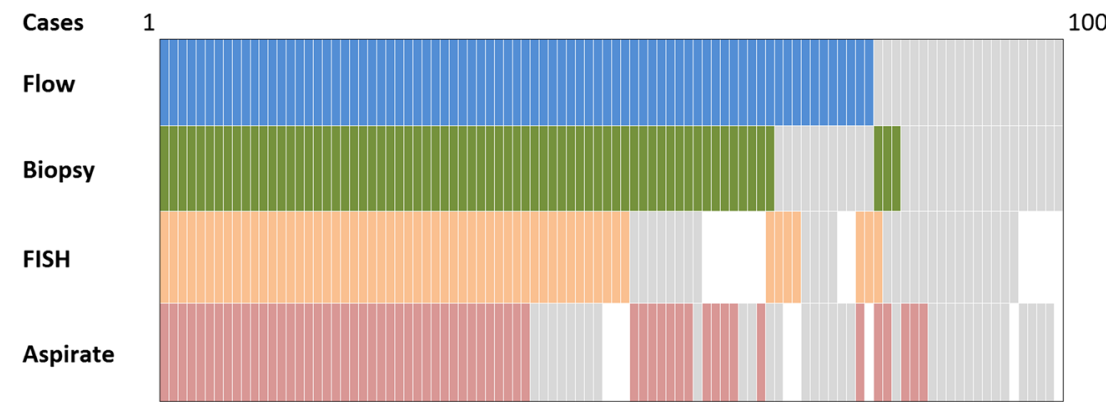

Fig. 2 Comparison of different testing modalities, BM CD138 IHC, aspirate smear counts, myeloma-specific interphase FISH, and FC in the 100 samples. Each vertically stacked box represents one sample. The blue, green, orange, and red boxes represent cases positive for PC neoplasm with different testing modalities. Gray boxes represent cases negative for PC neoplasm with different testing modalities. White boxes represent cases that were not tested with respective method 
Fig. 3 Correlation of percentage of plasma cells by BM CD138 IHC and aspirate smear count $(n=92)(p=0.001)$

Fig. 4 Analysis of plasma cells by four-color flow cytometry (FC). a The cells with dim to negative expression of CD45 and bright CD38 (green dots) are the neoplastic PCs. b, c These PCs also express CD138 but exhibit aberrant expression of CD56 and CD20 (partial). d, e The PCs demonstrate cytoplasmic kappa light chain restriction when compared with lambda. FITC fluorescein isothiocyanate, $P E$ phycoerythrin, $P E-C y 5.5$ PE-cyanine 5.5, $A P C$ allophycocyanin. $P 2$ gate represents plasma cells with normal phenotype

\section{Bone Marrow IHC versus Aspirate counts}

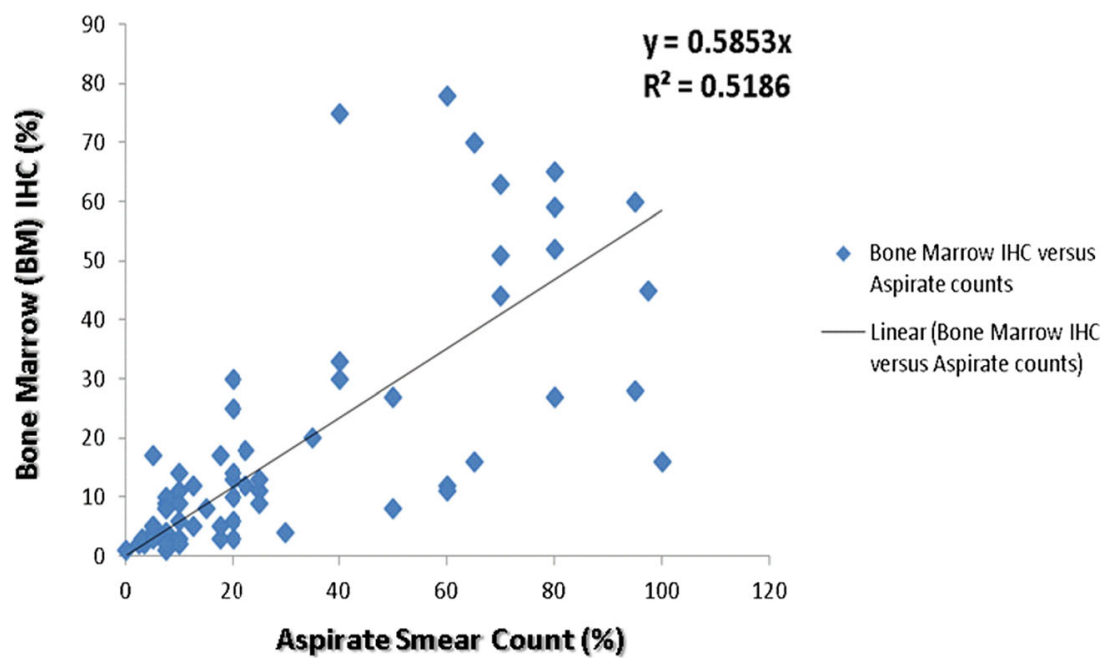

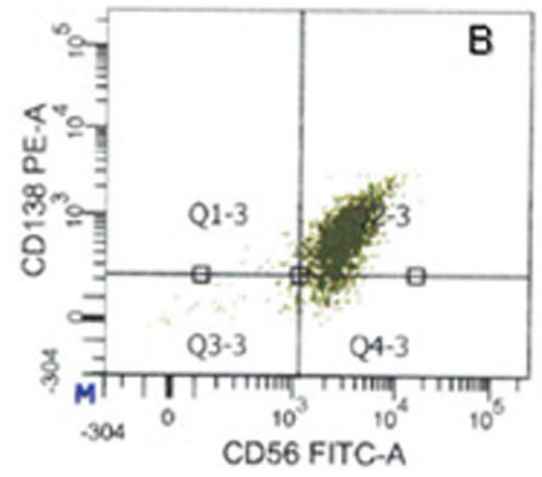
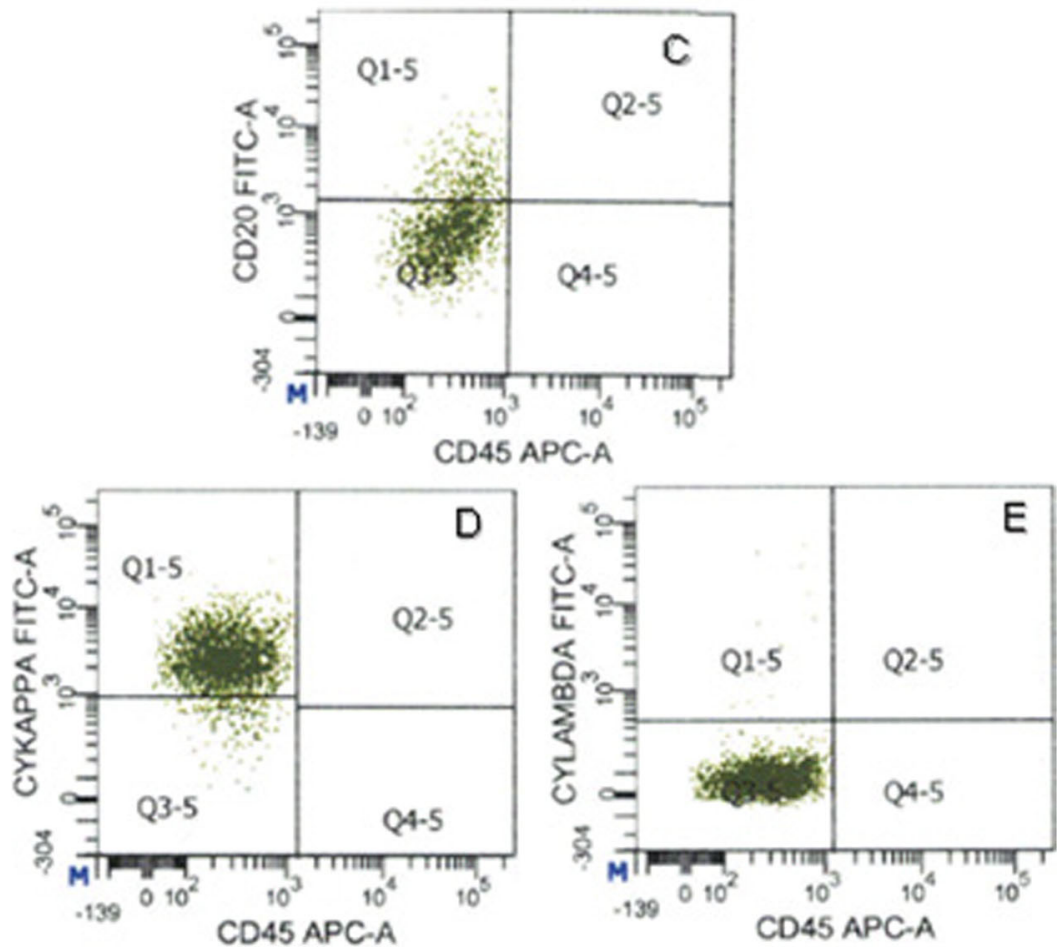
Fig. 5 FC of bone marrow PCs in a patient with $3 \%$ normal plasma cells on BM CD138 stain and aspirate count but with aberrancies seen by FCM. PCs were selected by gating on low dim to negative CD45 and bright CD38-positive events. a The abnormal PC population is highlighted by green dots and is negative for CD45. b The abnormal PCs (red circle) express CD138 while negative for CD19. c, $\mathbf{d}$ In addition, the PCs show aberrant expression of CD56 and CD117. d, e Lastly, the PCs show cytoplasmic lambda light chain restriction when compared with kappa. FITC fluorescein isothiocyanate, $P E$ phycoerythrin, $P E$-Cy5.5 PE-cyanine 5.5, APC allophycocyanin. $P 2$ gate represents plasma cells with normal phenotype; $P 3$ gate represents plasma cells with abnormal phenotype
Fig. 6 Correlation of percentage of plasma cells by FC analysis and BM CD138 IHC $(n=100)$ $(p=0.24)$
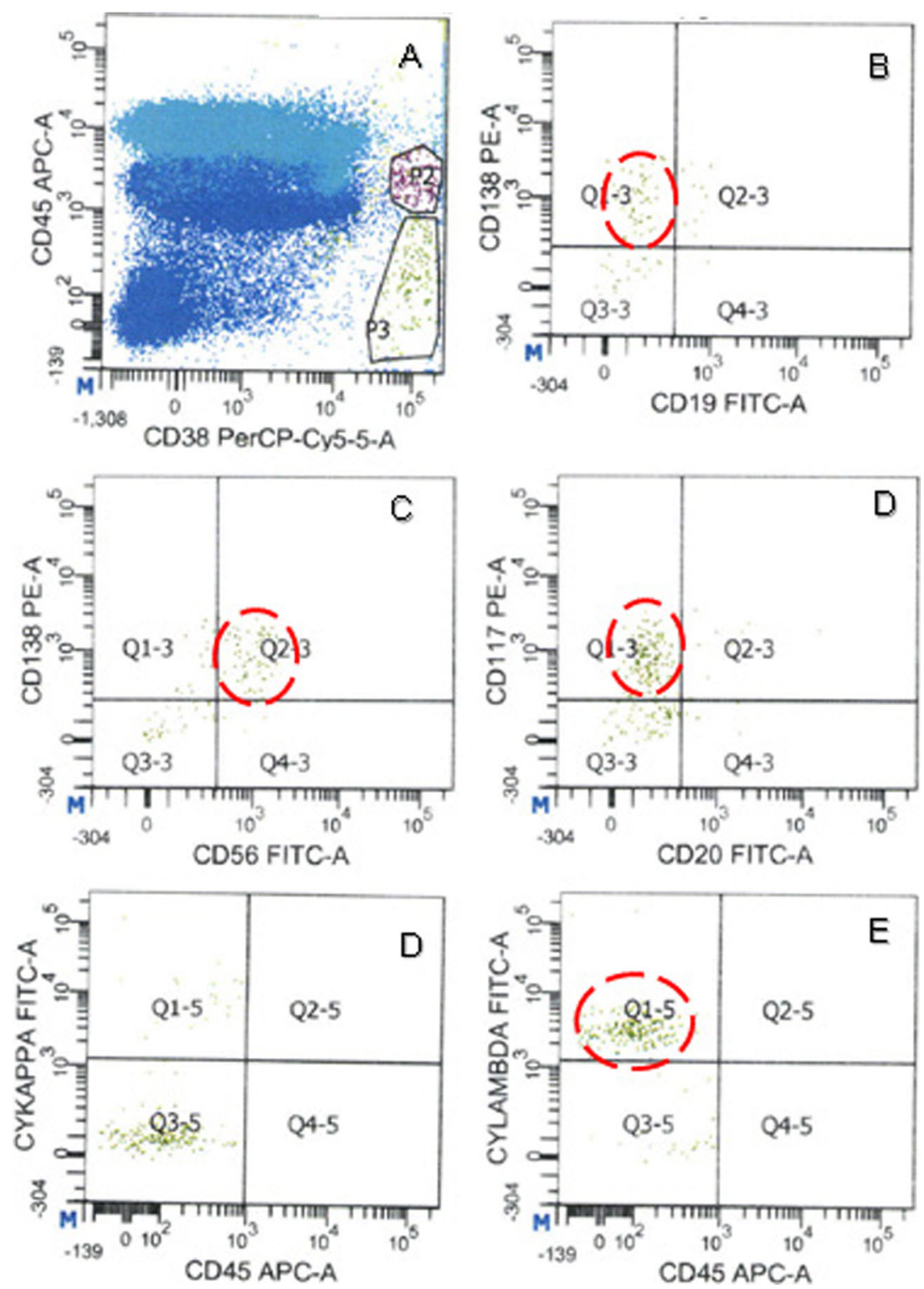

\section{$B M I H C$ versus $F C$}

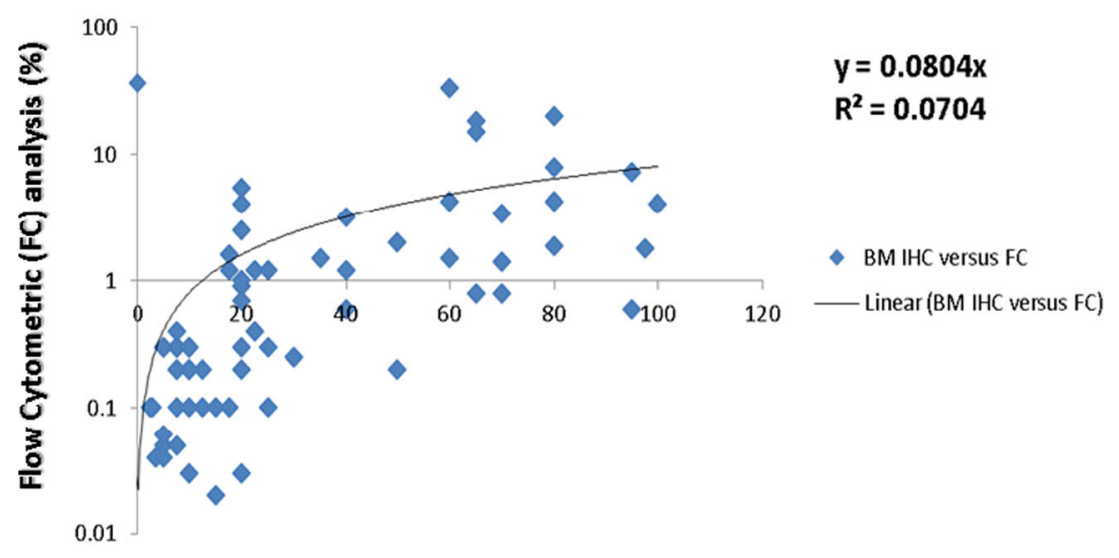

Bone marrow (BM) IHC (\%) 


\section{Assessment of plasma cell burden by FISH analysis}

The findings are summarized in Table 3. MM FISH/ cytogenetics was not as sensitive as FC or BM CD138 IHC $80 \%(59 / 75)$. However, MM FISH analysis had a $100 \%$ positive predictive value in the 100 samples analyzed. Of the positive cases, the most common FISH abnormality were aberrancies involving chromosome 13 followed by an increase in MLL. All nine patients with repeat samples had recurrent genetic aberrations by FISH analysis. Some patients (four of nine patients $44 \%$ ) had additional aberrations on follow-up samples. This likely represents progression in such cases.

\section{Discordant cases}

Ninety-three samples had concordant findings of the biopsy/ IHC and flow cytometry (concordance rate of $93 \%$ ). Five samples were positive by flow but negative on the biopsy/ IHC. These discordant cases all had $<5 \%$ plasma cells on the biopsy. We had concurrent MM FISH analysis on four of the five discordant cases. FISH results showed that three of the four cases $(75 \%)$ were also positive on FISH analysis. This supports the findings that flow is more sensitive in the establishment of residual disease. There were two cases that were positive by biopsy/IHC while negative by flow. Flow reports on these samples indicated significant clotting that yielded suboptimal flow samples.

\section{Discussion}

Identification of neoplastic plasma cells in the bone marrow is important for the accurate diagnosis and disease monitoring in patients with a PC neoplasm. The utility of different testing

Table 3 Myeloma-specific FISH findings $(n=86)$

\begin{tabular}{ll}
\hline Abnormal FISH (\%) & $59(69 \%)$ \\
$\mathrm{T}(4 ; 14)$ & $10(12 \%)$ \\
$\mathrm{T}(11 ; 14)$ & $11(13 \%)$ \\
$\mathrm{T}(8 ; 14)$ & $1(1 \%)$ \\
$1 \mathrm{q} 25$ (increase) & $(17 \%)$ \\
CBFB & $13(15 \%)$ \\
Del(13q) & $6(7 \%)$ \\
Monosomy 13 & $26(30 \%)$ \\
Del(17p) & $11(13 \%)$ \\
MLL (increase) & $27(31 \%)$ \\
ETV6 (increase) & $5(6 \%)$ \\
Del ETV6 & $4(5 \%)$ \\
High risk: any $\mathrm{t}(4 ; 14), \mathrm{t}(14 ; 16)$, or del $(17 \mathrm{p})$ & $17(20 \%)$ \\
\hline
\end{tabular}

modalities such as morphology combined with CD138 immunostain, aspirate PC count, flow cytometric analysis, and myeloma-specific FISH has not been well established. In this study, we evaluated the utilization of different testing modalities and demonstrated that IHC for CD138 is the most sensitive method for assessment of $\mathrm{PC}$ numbers in the $\mathrm{BM}$ specimens involved by a PC neoplasm. In contrast, FC immunophenotyping appears to be the most sensitive method to establish the clonal nature of the BM PC. In contrast, our data indicate that FISH analysis on CD138 selected PC does not offer additional information for disease monitoring but may be useful when there is a question of clinical progression by acquisition of high-risk genetic abnormalities such as $17 \mathrm{p}$ deletions or MYC region gene rearrangements.

\section{Methodological/analytical considerations}

\section{Immunohistochemistry}

The morphology of plasma cells in the BM biopsy can be difficult to assess on routine hematoxylin and eosin (H\&E) stains due to its resemblance to myeloid progenitors, and therefore, CD138 is extremely helpful in detecting the plasma cells. CD138 (syndecan-1) is a member of the syndecan family that comprises heparan sulfate proteoglycans. CD138 is significant for cell-cell and cell-matrix interactions. In adult human tissues, CD138 is predominantly expressed in epithelial cells and plasma cells. Of importance, within the BM microenvironment, it was found to be a specific marker for plasma cells and was not present in association with other hematopoietic cells or endothelial cells [10].

Our data shows that the use of CD138 on trephine bone marrow specimens yielded the highest enumeration of plasma cell burden when compared to other testing modalities. PC neoplasms are often focal (with small PC clusters or microaggregates), a fact that impacts the accuracy and reliability of the results of bone marrow plasma cell percentages obtained by differential counts of bone marrow aspirate smears. In addition, sampling differences between BM biopsy and aspirate specimens most likely explains the differences observed between the use of IHC and FCM results.

This supports previous data which suggests that CD138 is a highly sensitive and specific marker which is useful for the rapid and precise localization of normal and neoplastic PCs on routine BM sections [11]. Other markers such as IRF4/MUM1 [12] and VS38c [13] have also been shown to be specific for plasma cell detection in formalin-fixed paraffin-embedded specimens. However, the role of these markers in monitoring in plasma cell neoplasms has not been investigated.

Quantitation of bone marrow plasma cell percentage by the visual estimation of plasma cells using CD138 cell membrane staining has been suggested to give a higher estimation of the true plasma cell burden due to a larger area of bone marrow 
space occupied by PCs. Hence, other methods of PC enumeration such as multiple myeloma oncogene-1 (MUM-1), which exhibits nuclear staining, have been proposed to give a better estimate. However, MUM1 protein is expressed in a wide spectrum of hematolymphoid cells, decreasing its specificity in routine bone marrow specimens when compared to CD138 [12]. Plasma cell percentages obtained with CD138 sections have been previously shown to have the highest inter-observer concordance when compared with aspirate counts [14]. However, the reproducibility of PC percentage counts in itself by various observers is not well established. We suggest careful consideration of the above-suggested limitations of CD138 stain when assessing the percentage of plasma cell burden.

\section{Aspirate counts}

Our data reveals that BM CD138 IHC consistently revealed greater numbers of plasma cells in the bone marrow compared to aspirate counts as previously reported [9]. This difference can be explained by various pre-analytical and analytical variables including poorly representative, hemodiluted bone marrow aspirate specimens, clotting, sampling variability secondary to focal disease distribution, and inter-observer variability among others [9].

In addition, we found that $\mathrm{PC}$ enumeration by aspirate counts was less sensitive when compared to FISH analysis in the determination of bone marrow involvement by neoplastic plasma cells. This may be explained partly due to the enrichment of plasma cells using CD138 selection prior to performing the myeloma-specific interphase FISH procedure.

Assessment of plasma cell cytomorphology is best appreciated on the aspirate smears when compared to the H\&E section and/or CD138 IHC staining. Morphologic evaluation of PCs on the bone marrow trephine biopsy is best performed when the plasma cell burden is moderately to markedly increased, but at lower PC burden, the morphology is not well appreciated due to the presence of background myeloid precursors.

\section{Flow cytometric analysis}

$\mathrm{FC}$ is a multiparametric technique that has proven to be useful in the diagnosis of hematopoietic neoplasms as well as monitoring for minimal residual disease in the post-therapy setting [15]. Our data indicates that plasma cell enumeration using four-color FC analysis demonstrated the lowest percentage of plasma cell numbers when compared to the other modalities. This is in line with previous studies which showed that despite the use of sensitive techniques, FCM usually identifies fewer plasma cells than paraffin section CD138 IHC of biopsy sections [16]. The reason for the wide differences in plasma cell enumeration by conventional morphology combined with CD138 IHC and aspirate PC counts versus FCM is multifactorial. It has been attributed to the heterogeneous and often patchy pattern of bone marrow plasma cell infiltration in PC disorders and hence likely reflects sampling differences [17-19]. FCM is performed on the BM aspirate fluid which is depleted of lipid-adhesive PC [17] and shows greater contamination with peripheral blood. Both factors may also contribute to the lower PC counts and the limited diagnostic value of FCM PC counts $[9,17,18]$. In addition, plasma cells appear to be more easily lost during processing. Lastly, at MSKCC, the trephine bone marrow biopsy and aspirate sample are obtained earlier (first pull) whereas the aspirate material obtained for FCM is usually obtained from later aspirations or pulls and can often be of poor quality and not representative. In contrast, our data shows that FCM is the most sensitive method to establish the clonal nature of the BM PC when compared to other modalities.

\section{FISH}

The enrichment of plasma cells after separation of CD138+ cells prior to the performance of myelomaspecific interphase FISH helped to increase the sensitivity of the FISH test; however, the sensitivity of other testing modalities, e.g., FCM and CD138 IHC staining, was still higher. This greatly limits the use of myeloma-specific FISH in the routine follow-up of patients with an established PC neoplasm/myeloma. We observed a persistence of the similar cytogenetic abnormalities in a great majority of the repeated samples from the same patients. This supports the past observation that genetic abnormalities detected by cytogenetics/FISH analysis at the time of diagnosis can be used in the prognosis and risk stratification for guiding treatment in PC neoplasm/myeloma patients [3].

Previous studies have shown a loss of CD138 in samples stored in a cold environment (refrigerator) or when sample processing was delayed at the initial stage of setting up the assay [20]. In addition, identical samples analyzed with different RBC lysis methods or incubation periods yielded variable results. Given the high success rate of FISH analysis, the loss of CD138 during processing is not a major practical issue for CD138 bead enrichment method.

\section{Discordant cases}

Two samples were positive on morphology while negative by FC. FC reports on these cases noted that FC samples were clotted. Clotted samples processed by flow cytometry usually yield negative and inaccurate results. Accordingly, some studies have proposed lysis of the clots with streptokinase in 
situations where additional samples cannot otherwise be obtained [21]. Five samples were positive by flow but negative on the biopsy/IHC. On further review, all five cases had less than $5 \%$ plasma cells both by IHC and on aspirate counts. The positive flow results on these five cases were also positive by FISH in $75 \%$ of the samples tested. The $\leq 5 \%$ plasma cells in the marrow required for the determination of complete response which was proposed by the international uniform response criteria for multiple myeloma may not be applicable in these cases which show definite involvement by the neoplastic plasma cell clone. We propose using the clonality established by flow for all such cases to determine the further management of these patients.

\section{Clinical considerations}

From clinical perspective, we suggest that plasma cell numbers/enumeration obtained by CD138 may be the best established method for determination of tumor burden. The assessment of minimal residual disease (MRD) levels is of great utility to monitor the effects of therapy and predict patient outcome. In addition, accurate monitoring of minimal disease is vital when attempting to increase duration of remission. Our data shows that FCM is more sensitive in detecting very small numbers of abnormal plasma cells than CD138 by IHC and hence is more applicable in the follow-up of patients with an established PC neoplasm for the determination of MRD status. This supports previous findings which suggest that patients who achieve a FCM complete remission at day 100 post-transplant have a significantly longer progressionfree and overall survival [22]. Cytogenetic analysis has a role in establishing prognosis at the time of initial diagnosis but no superior role in the monitoring of disease.

In conclusion, we recommend CD138 assessment of tumor burden with the use of flow cytometric analysis for the evaluation of an abnormal phenotype to establish clonality and MRD.

\section{Compliance with ethical standards}

Conflict of interest Oluyomi E Ajise, MD, declares that she has no conflict of interest.

Mikhail Roshal, MD PhD, declares that he has no conflict of interest. $\mathrm{Lu}$ Wang, MD PhD, declares that she has no conflict of interest.

Goutamie N Sukhram, BSc MPH, declares that she has no conflict of interest.

Katherine M Smith, BSc, declares that she has no conflict of interest. Peter Maslak, MD, declares that he has no conflict of interest.

Ahmet Dogan, MD PhD, declares that he has no conflict of interest.

Ethical approval All procedures performed in studies involving human participants were in accordance with the ethical standards of the institutional and/or national research committee and with the 1964 Helsinki Declaration and its later amendments or comparable ethical standards.

\section{References}

1. Kyle RA, Rajkumar SV (2008) Multiple myeloma. Blood 111(6): 2962-2972. doi:10.1182/blood-2007-10-078022

2. Jemal A, Siegel R, Ward E, Hao Y, Xu J, Murray T, Thun MJ (2008) Cancer statistics, 2008. CA: Cancer J Clin 58(2):71-96. doi:10. 3322/CA.2007.0010

3. Zhan F, Sawyer J, Tricot G (2006) The role of cytogenetics in myeloma. Leukemia 20(9):1484-1486. doi:10.1038/sj.leu. 2404330

4. Fonseca R, Blood E, Rue M, Harrington D, Oken MM, Kyle RA, Dewald GW, Van Ness B, Van Wier SA, Henderson KJ, Bailey RJ, Greipp PR (2003) Clinical and biologic implications of recurrent genomic aberrations in myeloma. Blood 101(11):4569-4575. doi: 10.1182/blood-2002-10-3017

5. Fonseca R, Bergsagel PL, Drach J, Shaughnessy J, Gutierrez N, Stewart AK, Morgan G, Van Ness B, Chesi M, Minvielle S, Neri A, Barlogie B, Kuehl WM, Liebisch P, Davies F, Chen-Kiang S, Durie BG, Carrasco R, Sezer O, Reiman T, Pilarski L, Avet-Loiseau H (2009) International Myeloma Working Group molecular classification of multiple myeloma: spotlight review. Leukemia 23(12): 2210-2221. doi:10.1038/leu.2009.174

6. Munshi NC, Anderson KC, Bergsagel PL, Shaughnessy J, Palumbo A, Durie B, Fonseca R, Stewart AK, Harousseau JL, Dimopoulos M, Jagannath S, Hajek R, Sezer O, Kyle R, Sonneveld P, Cavo M, Rajkumar SV, San Miguel J, Crowley J, Avet-Loiseau H (2011) Consensus recommendations for risk stratification in multiple myeloma: report of the International Myeloma Workshop Consensus Panel 2. Blood 117(18):4696-4700. doi:10.1182/blood-2010-10300970

7. Durie BG, Harousseau JL, Miguel JS, Blade J, Barlogie B, Anderson K, Gertz M, Dimopoulos M, Westin J, Sonneveld P, Ludwig H, Gahrton G, Beksac M, Crowley J, Belch A, Boccadaro M, Cavo M, Turesson I, Joshua D, Vesole D, Kyle R, Alexanian R, Tricot G, Attal M, Merlini G, Powles R, Richardson P, Shimizu K, Tosi P, Morgan G, Rajkumar SV, International Myeloma Working G (2006) International uniform response criteria for multiple myeloma. Leukemia 20(9):1467-1473. doi:10.1038/sj. leu. 2404284

8. Rajkumar SV, Harousseau JL, Durie B, Anderson KC, Dimopoulos M, Kyle R, Blade J, Richardson P, Orlowski R, Siegel D, Jagannath $\mathrm{S}$, Facon T, Avet-Loiseau H, Lonial S, Palumbo A, Zonder J, Ludwig H, Vesole D, Sezer O, Munshi NC, San Miguel J, International Myeloma Workshop Consensus P (2011) Consensus recommendations for the uniform reporting of clinical trials: report of the International Myeloma Workshop Consensus Panel 1. Blood 117(18):4691-4695. doi:10.1182/blood-2010-10-299487

9. Ng AP, Wei A, Bhurani D, Chapple P, Feleppa F, Juneja S (2006) The sensitivity of CD138 immunostaining of bone marrow trephine specimens for quantifying marrow involvement in MGUS and myeloma, including samples with a low percentage of plasma cells. Haematologica 91(7):972-975

10. Bayer-Garner IB, Sanderson RD, Dhodapkar MV, Owens RB, Wilson CS (2001) Syndecan-1 (CD138) immunoreactivity in bone marrow biopsies of multiple myeloma: shed syndecan-1 accumulates in fibrotic regions. Mod Pathol 14(10):1052-1058. doi:10. 1038/modpathol.3880435

11. Chilosi M, Adami F, Lestani M, Montagna L, Cimarosto L, Semenzato G, Pizzolo G, Menestrina F (1999) CD138/syndecan1: a useful immunohistochemical marker of normal and neoplastic plasma cells on routine trephine bone marrow biopsies. Mod Pathol 12(12):1101-1106

12. Natkunam Y, Warnke RA, Montgomery K, Falini B, van De Rijn M (2001) Analysis of MUM1/IRF4 protein expression using tissue 
microarrays and immunohistochemistry. Mod Pathol 14(7):686694. doi:10.1038/modpathol.3880373

13. Turley H, Jones M, Erber W, Mayne K, de Waele M, Gatter K (1994) VS38: a new monoclonal antibody for detecting plasma cell differentiation in routine sections. J Clin Pathol 47(5):418-422

14. Al-Quran SZ, Yang L, Magill JM, Braylan RC, Douglas-Nikitin VK (2007) Assessment of bone marrow plasma cell infiltrates in multiple myeloma: the added value of CD138 immunohistochemistry. Hum Pathol 38(12):1779-1787. doi:10.1016/j.humpath.2007.04.010

15. Craig FE, Foon KA (2008) Flow cytometric immunophenotyping for hematologic neoplasms. Blood 111(8):3941-3967. doi:10.1182/ blood-2007-11-120535

16. Smock KJ, Perkins SL, Bahler DW (2007) Quantitation of plasma cells in bone marrow aspirates by flow cytometric analysis compared with morphologic assessment. Arch Pathol Lab Med 131(6): 951-955. doi:10.1043/1543-2165(2007)131[951:qopcib]2.0.co;2

17. Nadav L, Katz BZ, Baron S, Yossipov L, Polliack A, Deutsch V, Geiger B, Naparstek E (2006) Diverse niches within multiple myeloma bone marrow aspirates affect plasma cell enumeration. Br J Haematol 133(5):530-532. doi:10.1111/j.1365-2141.2006.06068.x

18. Rawstron AC, Orfao A, Beksac M, Bezdickova L, Brooimans RA, Bumbea H, Dalva K, Fuhler G, Gratama J, Hose D, Kovarova L, Lioznov M, Mateo G, Morilla R, Mylin AK, Omede P, PellatDeceunynck C, Perez Andres M, Petrucci M, Ruggeri M,
Rymkiewicz G, Schmitz A, Schreder M, Seynaeve C, Spacek M, de Tute RM, Van Valckenborgh E, Weston-Bell N, Owen RG, San Miguel JF, Sonneveld P, Johnsen HE (2008) Report of the European Myeloma Network on multiparametric flow cytometry in multiple myeloma and related disorders. Haematologica 93(3):431-438. doi: 10.3324/haematol.11080

19. San Miguel JF, Gutierrez NC, Mateo G, Orfao A (2006) Conventional diagnostics in multiple myeloma. Eur J Cancer 42(11):1510-1519. doi:10.1016/j.ejca.2005.11.039

20. Lin P, Owens R, Tricot G, Wilson CS (2004) Flow cytometric immunophenotypic analysis of 306 cases of multiple myeloma. Am J Clin Pathol 121(4):482-488. doi:10.1309/74r4-tb90-buwh-27jx

21. De Vis J, Renmans W, Segers E, Jochmans K, De Waele M (1991) Flow cytometric immunophenotyping of leukemia cells in clotted blood and bone marrow. J Immunol Methods 137(2):193-197

22. Paiva B, Vidriales MB, Cervero J, Mateo G, Perez JJ, Montalban MA, Sureda A, Montejano L, Gutierrez NC, Garcia de Coca A, de Las HN, Mateos MV, Lopez-Berges MC, Garcia-Boyero R, Galende J, Hernandez J, Palomera L, Carrera D, Martinez R, de la Rubia J, Martin A, Blade J, Lahuerta JJ, Orfao A, San Miguel JF (2008) Multiparameter flow cytometric remission is the most relevant prognostic factor for multiple myeloma patients who undergo autologous stem cell transplantation. Blood 112(10):4017-4023. doi:10.1182/blood-2008-05-159624 